Research Article

\title{
Music Education to Rescue Psychological Stress in Social Crisis Based on Fuzzy Prediction Algorithm
}

\author{
Jian Wang $(\mathbb{D}$ \\ Beihua University, Jilin, China \\ Correspondence should be addressed to Jian Wang; bhyy7777@126.com
}

Received 31 August 2021; Accepted 8 October 2021; Published 25 October 2021

Academic Editor: Punit Gupta

Copyright ( 92021 Jian Wang. This is an open access article distributed under the Creative Commons Attribution License, which permits unrestricted use, distribution, and reproduction in any medium, provided the original work is properly cited.

In order to alleviate human drowsiness in social crisis, it is necessary to predict the psychological rescue function of music education in social crisis and improve the accurate monitoring and analysis ability of the psychological rescue function of music education in social crisis; this paper puts forward a prediction model of psychological rescue function of music education in social crisis based on quantitative statistical analysis. The prediction and control model of the psychological rescue function of music education in social crisis is constructed, the fuzzy prediction algorithm is combined, the psychological rescue function characteristics of music education in social crisis are analyzed, and the descriptive statistical analysis model of the psychological rescue function of music education in social crisis is established. Through the fuzzy feature extraction method, the big data feature detection of the psychological rescue function of music education in social crisis is carried out, the statistical analysis model of the psychological rescue function of music education in social crisis is established, the dynamic analysis and prediction of the psychological rescue function of music education in social crisis are carried out by combining fuzzy information mining and adaptive learning methods, and the dynamic feature mining of the psychological rescue function of music education in social crisis is carried out by adopting the quantitative statistical feature analysis method. Statistical characteristics of the psychological rescue function of music education in social crisis are established, dynamic monitoring and feature prediction according to the analysis of the psychological rescue function of music education in social crisis are carried out, the ambiguity prediction and feature optimization judgment ability of the psychological rescue function of music education in social crisis are improved, and accurate prediction of the psychological rescue function of music education in social crisis based on the optimization statistical analysis results is carried out. The simulation results show that the statistical analysis ability and fuzzy judgment ability of using this method to predict the psychological rescue function of music education in social crisis are better, which improves the pertinence and effectiveness of music education.

\section{Introduction}

In music education, it is necessary to combine the social crisis for the relief and suppression analysis of the psychological rescue function of music education and establish a statistical analysis model of the psychological rescue function of music education in the social crisis. Combined with quantitative statistical analysis methods, the prediction and analysis of the psychological rescue function of music education in social crises are constructed, and the fuzzy detection analysis model of the psychological rescue function of music education in social crises is constructed [1]. Through the load dynamic analysis method, the dynamic analysis of the psychological rescue function of music education in social crisis is carried out. Combining the methods of dynamic statistical analysis and big data analysis, the improvement and fuzzy dynamic analysis of music education in social crisis is carried out, which improves the output stability of music education in social crisis [2]. Combined with the method of ambiguity analysis, the statistical analysis model of big data is used to predict and analyze the psychological rescue function of music education in social crisis. The dynamic big data joint analysis method is used to extract the dynamic feature quantity of the psychological rescue function of music education in social crisis. The prediction and dynamic evaluation of the 
psychological rescue function of music education in a social crisis have been carried out, which has improved the stability of the psychological rescue function of music education in a social crisis. The research on the rescue function prediction and big data analysis methods of music education has attracted great attention [3].

The prediction of the bailout function of music education is based on the big data collection and feature analysis of the psychological rescue function of music education. Among the traditional methods, the dynamic prediction methods for the rescue function of music education in social crisis mainly include fuzzy feature analysis methods, correlation dimension statistical analysis methods, and dynamic response feature detection methods. Reference [4] proposed a method for predicting the psychological rescue function of music education in social crisis based on the SSA-PPR model. It used the SSA-PPR model to build a big data statistical analysis model for the prediction of the psychological rescue function of music education in social crises and combined fuzzy information detection methods to achieve the psychological rescue function prediction of music education. This method improves the prediction accuracy of the rescue function of music education, but the prediction of the rescue function of music education by this method is ambiguous and has poor correlation. Reference [5] proposed a function prediction model based on descriptive statistical analysis and performed a change prediction and dynamic analysis of the psychological rescue function of music education in a social crisis. The dynamic feature quantity reflecting the psychological rescue function of music education in social crisis is extracted, and the method of correlation feature analysis is used to perform dynamic analysis and optimal scheduling of the psychological rescue function of music education in social crisis. This method has a relatively high degree of ambiguity in the dynamic analysis of the changes in the psychological rescue function of music education during social crises, and its ability to identify features is not good [6].

In order to solve the above problems, this paper proposes a prediction model for the psychological rescue function of music education in social crisis based on quantitative statistical feature analysis. The big data feature detection of the psychological rescue function of music education in social crisis is carried out by the method of fuzziness feature extraction. The quantitative statistical feature analysis method is used to mine the dynamic features of the psychological rescue function of music education in social crisis and establish the statistical feature quantity of psychological rescue function of music education in social crisis. In the simulation experiment, combined with the descriptive statistical analysis method, the rescue function prediction of music education in social crisis is realized.

\section{Statistical Analysis of Big Data of the Rescue Function of Music Education in Social Crisis}

2.1. Flow Sequence Modeling of the Rescue Function of Music Education in Social Crisis. In order to realize the prediction of the psychological rescue function of music education in social crisis, the SSA-PPR model is used to build a statistical analysis model of big data for the prediction of psychological rescue function of music education in social crisis. A descriptive statistical analysis method was used to establish the characteristic sequence distribution model of the psychological rescue function of music education in social crisis and to analyze the correlation feature of the psychological rescue function of music education in social crisis. The predictive feature quantity of the psychological rescue function of music education in social crisis is extracted, and a vertical topological analysis model of psychological rescue function of music education in social crisis is constructed. Through yield response control and fuzzy parameter identification methods, the longitudinal characteristics of the psychological rescue function of music education in social crisis are analyzed [7-9]. Combined with the fuzzy information correlation prediction method, the rescue function prediction model of music education in social crisis is obtained, and the longitudinal distribution sequence of rescue function of music education in social crisis is as follows:

$$
A(x)=A J(x) a(x)+B(1-b(x))
$$

$\left\{\begin{array}{l}\dot{m}_{i}(t)=-a_{i} m_{i}(t)+b_{i}\left(p_{i}(t-\sigma), p_{2}(t-\sigma), \cdots, p_{n}(t-\sigma)\right) \\ \dot{p}_{i}(t)=-c_{i} p_{i}(t)+d_{i} m_{i}(t-\tau)\end{array}\right.$ $p_{i}(t)=-c_{i} p_{i}(t)+d_{i} m_{i}(t-\tau)$

is a set of dynamic distribution features of the psychological rescue function of music education in social crisis. $h(t)=$ $H \sum_{m=1}^{M} \sum_{k=1}^{K(m)} \alpha_{m k} \delta\left(t-T_{m}-\tau_{m k}\right)$ is the load intensity distribution set of the psychological rescue function of music education in social crisis. The attribute value of the psychological rescue function of music education in the social crisis of $V=\left\{v_{1}, v_{2}, \cdots, v_{N}\right\}$ is $(u, v) \in E$. In the prediction process of the psychological rescue function of music education in a social crisis, a directed graph analysis model $h(t)=H \sum_{m=1}^{M} \sum_{k=1}^{K(m)} \alpha_{m k} \delta\left(t-T_{m}-\tau_{m k}\right)$ is used to represent the statistical distribution of the psychological rescue function of music education in a social crisis where $V$ is the autocorrelation distribution set of the psychological rescue function of music education in social crisis, $V=\left\{v_{1}, v_{2}, \cdots, v_{N}\right\}$, and the vertical imbalance dynamic distribution set of the psychological rescue function of music education in social crisis is $q_{i}(t 1)=[w 1, x 1, y 1, z 1]$ and $q_{i}(t 2)=[w 2, x 2, y 2, z 2]$. Calculating the feature quantity of the plane motion state of the psychological rescue function of music education in the social crisis, the feature 
distribution set is $(u, v) \in E$, and $W=\left\{\omega_{1}, \omega_{2}, \cdots, \omega_{m}\right\}$ is the dynamic weight of the psychological rescue function of music education in a social crisis. Using high-dimensional phase space reconstruction technology to carry out spatial reorganization of the psychological rescue function sequence of music education in social crisis, the reconstructed phase space is as follows:

$$
\begin{aligned}
\mathbf{X} & =\left[\mathbf{x}\left(t_{0}\right), \mathbf{x}\left(t_{0}+\Delta t\right), \cdots, \mathbf{x}\left(t_{0}+(K-1) \Delta t\right)\right] \\
& =\left[\begin{array}{cccc}
x\left(t_{0}\right) & x\left(t_{0}+\Delta t\right) & \cdots & x\left(t_{0}+(K-1) \Delta t\right) \\
x\left(t_{0}+J \Delta t\right) & x\left(t_{0}+(J+1) \Delta t\right) & \cdots & x\left(t_{0}+(K-1) \Delta t+J \Delta t\right) \\
\vdots & \cdots & \ddots & \cdots \\
x\left(t_{0}+(m-1) J \Delta t\right) & x\left(t_{0}+(1+(m-1) J) \Delta t\right) & \cdots & x\left(t_{0}+(N-1) \Delta t\right)
\end{array}\right],
\end{aligned}
$$

where $\mathbf{x}(t)$ is the dynamic feature distribution set of the psychological rescue function of music education in social crisis, $J$ is the disturbance window function, $m$ is the embedding dimension of the psychological rescue function of music education in social crisis, and $\Delta t$ is the jump width of psychological rescue function of music education in social crisis. The probability density of the state distribution of the psychological rescue function state of music education in the social crisis is as follows:

$$
w_{i j}=\beta \times w\left(e_{p} k_{q}\right)(\beta>1),
$$

where $\beta$ is a positive definite periodic solution and $w\left(e_{p} k_{q}\right)$ is the fluctuation coefficient of the psychological rescue function of music education in a social crisis. Combined with the fuzzy prediction algorithm, the characteristic analysis of the psychological rescue function of music education in social crisis is carried out, and a descriptive statistical analysis model of the psychological rescue function of music education in social crisis is established. Through the fuzziness feature extraction method, the big data feature detection of the rescue function of music education in social crisis [10], the difference function is as follows:

$$
\widehat{W}=\left\{\begin{array}{ll}
\operatorname{sgn}(W)(|W|-\alpha T s) & |W| \geq T s \\
0 & |W|<T s
\end{array},\right.
$$

where $\alpha$ is the adaptive adjustment coefficient of the psychological rescue function of music education in social crisis and $W$ is the steady-state feature solution for the prediction of psychological rescue function of music education in social crisis, and its value range is $0 \leq \alpha \leq 1$. Based on the above analysis, a time series distribution model of the psychological rescue function of music education in social crises is established, and a dynamic analysis is performed based on the psychological rescue function distribution of music education in social crises [11].

2.2. Statistical Analysis of the Rescue Function of Music Education. The PCA model is used to build a big data statistical analysis model for the prediction of the psychological rescue function of music education in a social crisis. The SSA-PPR model function is as follows:

$$
\left.\begin{array}{l}
\min _{w, b, \xi} \frac{1}{2}\|w\|^{2}+C \sum_{j=1}^{l} u\left(x_{j}\right) \xi_{j} \\
\text { s.t. } y_{j}\left(\left(w \cdot x_{j}\right)+b\right)+\xi_{j} \geq 1 \\
\xi_{j} \geq 0, j=1,2, \ldots, l
\end{array}\right\} .
$$

Taking the main component features of the psychological rescue function of music education in social crisis as the reference feature quantity, the fourth-order Runge-Kutta method is used to solve the longitudinal imbalance feature quantity of psychological rescue function of music education in social crisis [12]. The kernel function of the model distribution for the psychological rescue function of music education is $k\left(x_{i}, x_{j}\right)$. Then, the linear programming function of the psychological rescue function of music education in the social crisis is as follows:

$$
\left.\begin{array}{l}
\min _{\alpha} \frac{1}{2} \sum_{i=1}^{l} \sum_{j=1}^{l} y_{i} y_{j} \alpha_{i} \alpha_{j} K\left(x_{i}, x_{j}\right)-\sum_{j=1}^{l} \alpha_{j} \\
\text { s.t. } \sum_{j=1}^{l} y_{j} \alpha_{j}=0 \\
0 \leq \alpha_{j} \leq u\left(x_{j}\right) C, \quad j=1,2, \ldots, l
\end{array}\right\}
$$

The linear programming design of the psychological assistance function of music education in social crises uses the square programming algorithm [13]. The optimized function for predicting the psychological assistance function of music education in social crises is as follows:

$$
h(t)=H \sum_{m=1}^{M} \sum_{k=1}^{K(m)} \alpha_{m k} \delta\left(t-T_{m}-\tau_{m k}\right) .
$$

To construct the topological distribution function of the psychological rescue function of music education in social 
crisis, the statistical analysis model of psychological rescue function of music education in social crisis is as follows:

$$
\frac{\partial u_{i}}{\partial p_{i}}=\frac{G h_{i}}{\sum_{j \neq i} h_{j} p_{j}+\sigma^{2}\left(1 / 1+\gamma_{i}-\beta_{c_{1}}\right)} .
$$

According to the results of statistical analysis, the characteristics of the psychological rescue function of music education in social crisis are decomposed, and the multidimensional scale feature distribution is obtained as follows:

$$
\eta_{\text {comm }}=\frac{k_{1} \cdot l}{E_{\text {comm }}} \cdot\left(1-p_{\text {drop }}\right),
$$

where $p_{\text {drop }}$ is the SSA-PPR model parameter of the psychological rescue function of music education in social crisis. Through the fuzziness feature extraction method, the big data feature detection of the psychological rescue function of music education in social crisis is established to establish a descriptive statistical analysis model of the psychological rescue function of music education in social crisis [14].

\section{Predictive Model of Rescue Function of Music Education in Social Crisis}

3.1. Big Data Analysis of the Rescue Function of Music Education in Social Crisis. This paper proposes a method for predicting the psychological rescue function of music education in the social crisis based on the SSA-PPR model. By extracting the feature analysis of the psychological rescue function of music education in social crisis [15], the distribution set of the statistical characteristics of the two-way planning to analyze the psychological rescue function of music education in social crisis is as follows:

$$
S_{b}=\sum_{i=1}^{c} p_{i}\left(\vec{m}_{i}-\vec{m}\right)\left(\vec{m}_{i}-\vec{m}\right)^{T},
$$

where $\vec{m}=\sum_{i=1}^{c} p_{i} \vec{m}_{i}$ is the autocorrelation adjustment component of the psychological rescue function of music education in social crisis. According to the correlation index of the psychological rescue function of music education in social crisis, $n$ variables of the psychological rescue function of music education in social crisis are collected. Taking the feature decomposition to get the association rule set of the psychological rescue function of music education in social crisis, expressed by $a_{j}$, the template function of the psychological rescue function prediction of music education in social crisis is described as follows:

$$
\left\{\begin{array}{l}
G_{1}=b_{11} a_{1}+b_{12} a_{2}+\cdots b_{1 n} a_{n} \\
G_{2}=b_{21} a_{1}+b_{22} a_{2}+\cdots b_{2 n} a_{n} \\
\cdots \quad \cdots \quad \cdots \\
G_{n}=b_{n 1} a_{1}+b_{n 2} a_{2}+\cdots+b_{n n} a_{n}
\end{array}\right.
$$

Both $G_{j}$ and $G_{k}$ have a strong correlation. $G_{k}$ represents the difference in the psychological rescue function of music education in social crisis. $G_{j}$ is the main component characteristic quantity of the psychological rescue function of music education in social crisis. The template matching method is used to obtain the adaptive weighting coefficient of the psychological rescue function of music education in social crisis, and the fuzzy information weighting matrix is as follows:

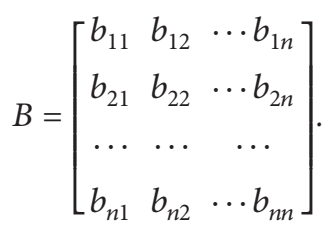

Using adaptive learning methods, the fitting coefficient of the psychological rescue function of music education in social crisis is $S=\left\{\left(x_{1}, y_{1}, u\left(x_{1}\right)\right), \ldots,\left(x_{l}, y_{l}, u\left(x_{l}\right)\right)\right\}$ where $x_{j} \in R^{n}, u\left(x_{j}\right) \in\{-1,1\}, \sigma \leq u\left(x_{j}\right) \leq 1, \sigma$ is the model parameter of the statistics of the psychological rescue function of music education in the social crisis and $u\left(x_{j}\right)$ is the psychological rescue function of music education in social crisis. The output of $\left(x_{j}, y_{j}, u\left(x_{j}\right)\right)$ is the correlation $(j=$ $1, \ldots, l$ ) of $y_{j}=1$ (positive category) or $y_{j}=-1$ (negative category). Combined with fuzzy information mining and adaptive learning methods, the dynamic analysis and prediction of the psychological rescue function of music education in social crisis are carried out.

3.2. Rescue Function of Music Education. According to the analysis of the psychological rescue function of music education in social crises, dynamic monitoring and feature prediction are carried out to establish the statistical characteristic quantity of psychological rescue function of music education in social crises. This improves the ability of ambiguity prediction and feature optimization judgment of the psychological rescue function of music education in social crisis. The fuzzy output feature set of music education is $\mathrm{CH}_{i}\left(i \in \mathrm{C}_{1}\right)$. Combined with the optimized statistical analysis results, the psychological relief effect of music education in social crisis was predicted accurately, and, the output is as follows:

$$
\begin{array}{r}
f(x)=\operatorname{sgn}\left\{\sum_{j=1}^{l} \alpha_{j}^{*} y_{j} K\left(x, x_{j}\right)+b^{*}\right\}, \\
x \in R^{n},
\end{array}
$$

where $b^{*}=y_{i}-\sum_{j=1}^{l} y_{j} \alpha_{j} K\left(x_{j}, x_{i}\right), i \in\left\{i \mid 0<\alpha_{i}^{*}<u\left(x_{i}\right) C\right\}$. The statistical feature quantity of the psychological rescue function of music education in social crisis is established, and dynamic analysis based on the fusion result of the psychological rescue function of music education in social crisis is performed. The characteristic quantity of ambiguity is as follows:

$$
Y_{k}=\left[y_{k 1}, y_{k 2}, \cdots, y_{k j}, \cdots, y_{k J}\right], \quad(k=1,2, \cdots, N),
$$

where $y_{k j}$ represents the unbalanced feature quantity of the psychological rescue function of music education in social crisis and $N$ is the data length of psychological rescue 


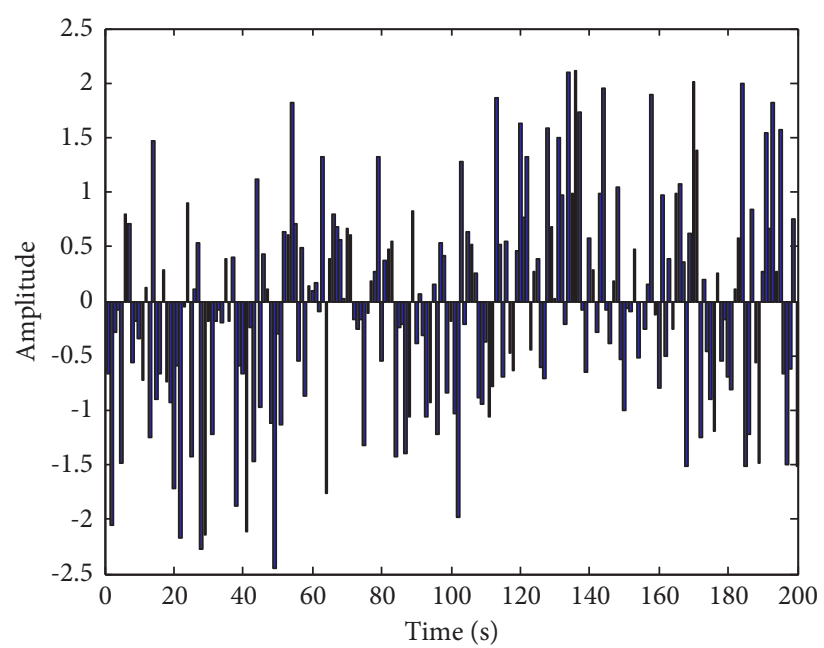

Figure 1: Time domain distribution of the psychological rescue function of music education in social crisis.

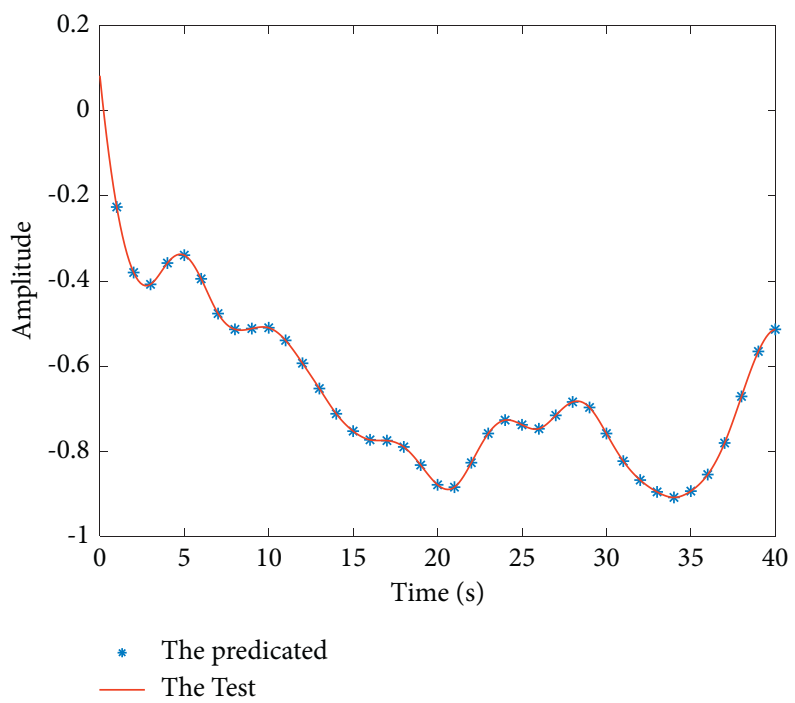

FIgUre 2: Rescue function index of music education in social crisis.

function of music education in social crisis. Through the association data mining, the optimization prediction and evaluation of the psychological rescue function of music education in social crisis are realized.

\section{Simulation Test and Analysis}

Through simulation experiments, the effectiveness of the method in this paper in realizing the prediction and quantitative analysis of the psychological rescue function of music education in social crisis is verified. The number of days of bailout guidance for social education in social crisis is 24 days, the length of the intermittent sample sampling is $1 \mathrm{~h}$, the number of samples for statistical analysis of the psychological rescue function of music education is 500 , the correlation coupling coefficient is 0.26 , the ambiguity coefficient is 0.28 , and the root mean square error is set to 0.25 . According to the above parameter settings, the rescue function information of music education in social crisis is sampled, and the large data distribution of the sampled samples is shown in Figure 1.

According to the sampling results of the psychological rescue function information of music education in the social crisis in Figure 1, the changes in the psychological rescue function of music education in the social crisis are predicted. Using the SSA-PPR model to build a big data statistical analysis model for the prediction of the rescue function of music education in social crises, the rescue function index of music education in social crises is obtained, as shown in Figure 2.

It can be seen from Figure 2 that the method in this paper can effectively predict the rescue function of music education in social crisis, and the prediction has good convergence. The prediction accuracy test was conducted, and the comparison results are shown in Table 1. It can be obtained that the method proposed in this paper has a higher precision in predicting the psychological rescue function of music education in social crisis. 
TABLE 1: Comparison of prediction accuracy of psychological rescue function of music education in social crisis.

\begin{tabular}{|c|c|c|c|c|}
\hline Number of iterations & This paper method & Reference [3] & Reference [4] & Reference [6] \\
\hline 100 & 0.945 & 0.856 & 0.878 & 0.878 \\
\hline 200 & 0.967 & 0.888 & 0.897 & 0.898 \\
\hline 300 & 0.980 & 0.890 & 0.902 & 0.895 \\
\hline 400 & 0.993 & 0.932 & 0.923 & 0.895 \\
\hline
\end{tabular}

\section{Conclusion}

In order to construct an ambiguity detection and analysis model for the psychological rescue function of music education in social crises, this paper combines the method of ambiguity analysis and uses the big data statistical analysis model to predict and analyze the psychological rescue function of music education in social crisis. This paper proposes a prediction model of the psychological rescue function of music education based on quantitative statistical characteristics analysis. It uses high-dimensional phase space reconstruction technology to carry out the spatial reorganization of the psychological rescue function sequence of music education in social crisis. A descriptive statistical analysis model for the psychological rescue function of music education in social crises is established, and the big data feature detection of the psychological rescue function of music education in social crises is carried out by fuzziness feature extraction. And combined with fuzzy information mining and adaptive learning methods, the dynamic analysis and prediction of the psychological rescue function of music education in social crisis are carried out. The study shows that the accuracy of the rescue function prediction of music education in social crises in this paper is high, and the feature matching is good.

\section{Data Availability}

Data sharing not applicable to this article as no datasets were generated or analyzed during the current study.

\section{Conflicts of Interest}

The author declares that there are no conflicts of interest with respect to the research, authorship, and/or publication of this article.

\section{Acknowledgments}

This paper is the result of the project of Jilin Provincial Department of Education: The Construction of Jilin Provincial College Students' Growth Planning Platform (jjkh20200093sk) and Jilin Provincial Education Science Planning Project: Research on the Relief Function of Music in Social Crisis (gh20283).

\section{References}

[1] J. Sun, Y. Wu, G. Cui, and Y. Wang, "Finite-time real combination synchronization of three complex-variable chaotic systems with unknown parameters via sliding mode control," Nonlinear Dynamics, vol. 88, no. 3, pp. 1677-1690, 2017.
[2] X. Chen, T. Huang, J. Cao, J. H. Park, and J. Qiu, "Finite-time multi-switching sliding mode synchronisation for multiple uncertain complex chaotic systems with network transmission mode," IET Control Theory \& Applications, vol. 13, no. 9, pp. 1246-1257, 2019.

[3] Y. Yu, Z. Y. Wang, and D. G. Xu, "Speed and current sensors fault detection and isolation based on adaptive observers for induction motor drivers," Journal of Power Electronics, vol. 5, no. 14, pp. 967-979, 2014.

[4] W. Zhang and Z. Wang, "Research on join operation of temporal big data in distributed environment," Computer Engineering, vol. 45, no. 3, pp. 20-25, 2019.

[5] Y. Goldberg, "A primer on neural network models for natural language processing," Journal of Artificial Intelligence Research, vol. 57, no. 1, pp. 345-420, 2016.

[6] H. Zhang, L. I. Chong, Y. Ke, and S. Zhang, "A distributed user browse click model algorithm," Computer Engineering, vol. 45, no. 3, pp. 1-6, 2019.

[7] D. Yu, X. Yuan, W. Zhang, and C. Wang, "Spatiotemporal crowdsourcing online task allocation algorithm based ondynamic threshold," Journal of Computer Applications, vol. 40, no. 3, pp. 658-664, 2020.

[8] T. S. Song, Y. X. Tong, L. B. Wang, and K. Xu, "Online task assignment for three types of objects under spatial crowdsourcing environment," Journal of Software, vol. 28, no. 3, pp. 611-630, 2017.

[9] U. ul Hassan and E. Curry, "Efficient task assignment for spatial crowdsourcing: a combinatorial fractional optimization approach with semi-bandit learning," Expert Systems with Applications, vol. 58, no. C, pp. 36-56, 2016.

[10] J. Wang, W. Liu, W. Xing, and S. Zhang, "Visual object tracking with multi-scale superpixels and color-feature guided kernelized correlation filters," Signal Processing: Image Communication, vol. 63, pp. 44-62, 2018.

[11] M. Danelljan, G. Häger, F. S. Khan, and M. Felsberg, "Discriminative scale space tracking," IEEE Transactions on Pattern Analysis and Machine Intelligence, vol. 39, no. 8, pp. 1561-1575, 2017.

[12] J. F. Henriques, R. Caseiro, P. Martins, and J. Batista, "Highspeed tracking with kernelized correlation filters," IEEE Transactions on Pattern Analysis and Machine Intelligence, vol. 37, no. 3, pp. 583-596, 2015.

[13] D. S. Bolme, J. R. Beveridge, B. A. Draper et al., "Visual object tracking using adaptive correlation filters," in Proceedings of the 2010 IEEE Conference on Computer Vision and Pattern Recognition, pp. 2544-2550, IEEE, San Francisco, CA, June 2010.

[14] X. Zhang and Y.-H. He, "Modifid interpolatory projection method for weakly singular integral equation eigenvalue problems," Acta Mathematicae Applicatae Sinica, English Series, vol. 35, no. 2, pp. 327-339, 2019.

[15] Z. Zhang Chaohua, L. Li, and G. Yun, "Study on moving dislocations in decagonal quasicrystals," Chinese Journal of Solid Mechanics, vol. 38, no. 2, pp. 165-169, 2017. 